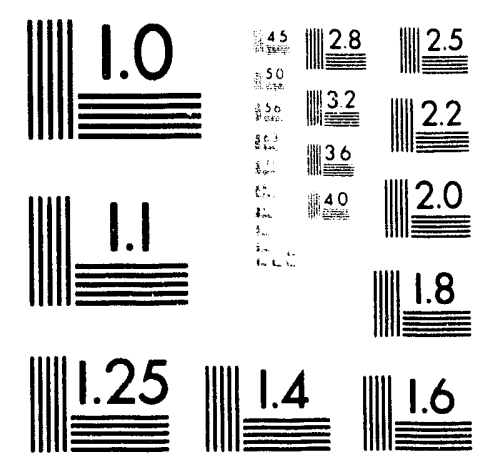



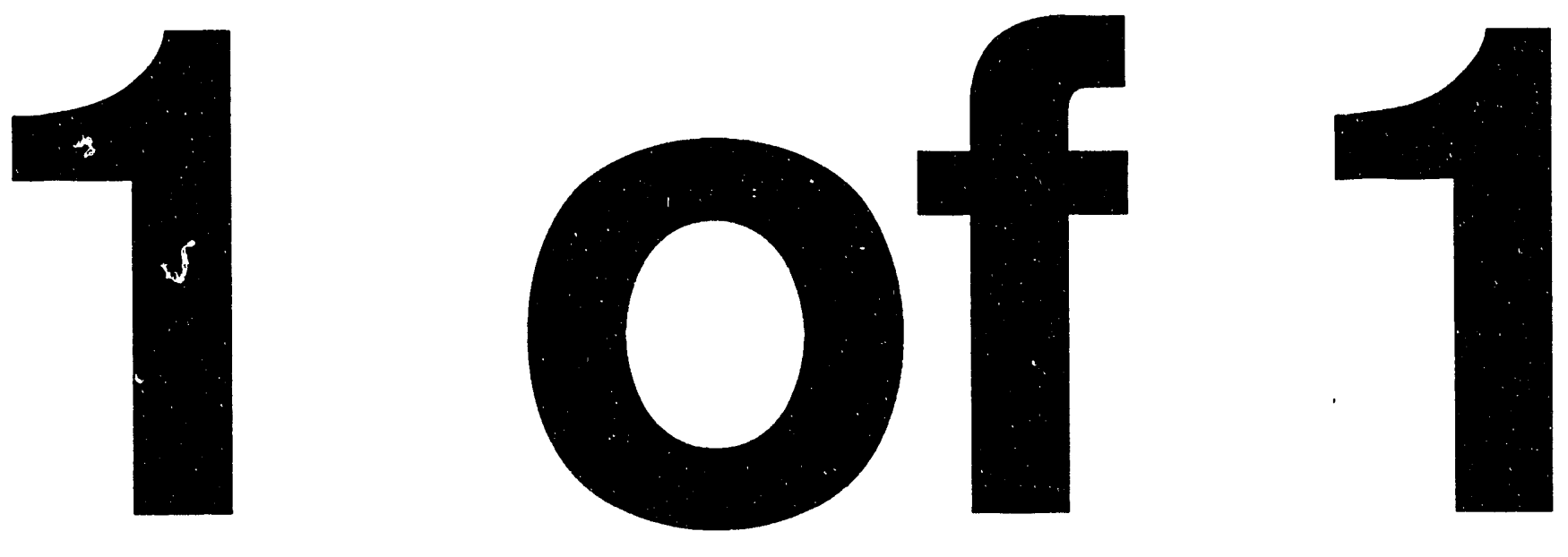
LBL-33284

UC-419

HIFAN-569

\title{
Electrostatic Quadrupoles for Heavy-Ion Fusion
}

\author{
P. Seidl and A. Faltens \\ Accelerator \& Fusion Research Division \\ Lawrence Berkeley Laboratory \\ University of California \\ Berkeley, California 94720
}

Submitted to the 1993 Particle Accelerator Conference

Washington, D. C., May 17 - 20, 1993

*This work was supported by the Office of Energy Research, Office of Fusion Energy, U. S. Department of Energy under Contract No. DE-AC03-76SF00098. 


\title{
Electrostatic Quadrupoles for Heavy-Ion Fusion ${ }^{*}$
}

\author{
Peter Seidl and Andy Faltens \\ Lawrence Berkeley Laboratory \\ Berkeley, CA 94720 USA
}

\section{Abstract}

Voltage-holding data for three quadrupole electrode sizes and inter-electrode spacings are reported. The dependence of the breakdown voltage on system size and its infuence on the optimum quadrupole size for beam transport in a multiple beam array are discussed.

\section{INTRODUCTION}

A number of designs for a heavy ion induction linac to be used as a driver for inertial confinement fusion (ICF) incorporate electrostatic quadrupoles as elements in an altematinggradient-focusing latuce in the low-energy portion. This arises from the efficiency of the electrostatic quadrupole, in particular at low ion velocity, when compared to the magnetic quadrupole. Important objectives in designing a driver in this application intended for commercial electricity generation are efficiency and cost minimization, and thus, optimization of the beam current transported along the accelerator. To accelerate the large charge necessary to drive ICF, about $400 \mu \mathrm{C} / \mathrm{pulse}$, it is advantagcous to use multiple parallel beams which are focused in individual transport channels but are accelerated by common induction modules. Other things being equal, the cost of the accelerator decreases as the volume occupied by this charge decreases. The advantages of electrostatic quadrupoles are low manufacturing cost, economical operation, and the ability to transport a large amount of charge. The primary disadvantage is the risk of electrical breakdown either between the quadrupole electrodes, across the insulating supports or at the high-voltage fecdthrough.

Figure 1 shows a schematic of the quadrupole geometry transverse to the beam axis. Field quality is an important issue, due to the necessity of maintaining low emittance so that beam combining and final focusing requirements may be achieved. In this work we have used the "ideal quadrupole geometry" which has circular cylindrical electrodes with $8 / 7$ of the aperture diameter for their diameter. With this ratio the fields in the overlap region are almost purely quadrupolar and the dodecapole component vanishes[1]. Longitudinally, the structure is interdigitated, shown in Fig. 3, where the quadrupole electrodes are cantilevered from opposite polarity end plates, and peripheral insulators hold the assembly together. The basic electrode structure in Figs. 1 and 3 is repeated in order to accommodate multiple beams in a single induction core. If perfect manufacturing and alignment were possible, then the optimum solution would be a very large number of microscopic beams -- the examination of this possibility was

\footnotetext{
"This work was supported by the Director, Office of Energy Research, Office of Fusion Energy, U.S. Dept. of Energy, under contract no. DE-AC03-76SF00098.
}

advocated by Maschke[3] with the Meqalac concept. Closely related to the question of the number of beams to be used is the beam-combining process. The geometric and space charge dilution of emiltance from combining and the low emittance required by the final focusing system limit the number of beam combinings to one or two. In the low energy section a very large number of beams is desired, whereas, in most of the accelerator, the lowest cost is found for a small number of beams. For final focusing, however, a larger number is desirable again. With the present undersianding of these matters, the driver would have about 80 electrostatically-focused beams in the front end, and 20 magnetically-focused beams in the remainder.

A common formulation of the beam transport is given by solutions to the Kapchinskij-Vladimirskij envelope equations, in terms of hard-edge equivalent fields with an occupancy of focusing fields, $\eta$, and a half period, $L$. Using the tables and equations of Ref. 2 in the limit of extreme space-charge dominance, we will establish a figure of merit (FOM) for transport in an clectrostatically-focused, multiple-bcam array that depends on the voltage holding characteristics of the quadrupoles, and the clearance limits of the beam edge to the physical aperture.

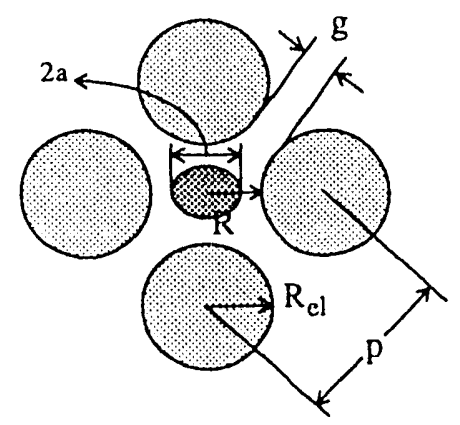

Figure 1. Schematic of electrode layout transverse to the beam direction. $p$ is the repeat size of the quadrupole array geometry, $a$ is the beam size, $g$ is the inter-electrode gap size, $R$ is the aperture radius and $R_{e l}$ is the electrode radius.

First, the longitudinal dimensions of the quadrupole determine the maximum $\eta$ available. At a low beam energy, the end plates and high-voltage clearance around them take up most of the "dead space", (1- $\eta) L$; this is a consequence of needing large radii of curvature around the beam holes. It appears that $10-13 \mathrm{~cm}$ of dead space are necessary; for example, this implies $\eta \approx 0.5$ and $\mathrm{L}=30 \mathrm{~cm}$ at $\mathrm{T}=2.5 \mathrm{McV}$.

The transportable line charge density, $\lambda$, is

$$
\lambda=2 \pi \varepsilon_{o} \gamma^{2} k V_{q}\left(\frac{a}{R}\right)^{2}
$$

where $a$ is the maximum beam radius, $R$ is the aperture radius and $\gamma=\left(1-\beta^{2}\right)^{-1 / 2}$ is the Lorente transformation parameter. The 
factor $k$ is a scaled interisity/(beam envelope area) parameter which is a monotonically increasing function of $\eta$ and the undepressed tune (single-particle, betatron phase advance per cell) $\sigma_{0}$. Beam stability considerations dictate a maximum allowable $\sigma_{0}$ of $85^{\circ}-90^{\circ}[4]$. The standard clearance prescription has been

$$
R=1.25 a+1(\mathrm{~cm})
$$

This is based on experiments [5] and simulations which showed undesirable image effects for high-intensity beams which filled more than about $80 \%$ of the aperture (the factor 1.25) and the expected growth with distance of coherent oscillations from misalignment, field errors and mismatches (the $+1 \mathrm{~cm} \mathrm{term}^{\dagger}$ ). With these constraints, it can be shown that $\lambda$ is an increasing function of $\mathrm{T}$, with the greatest increase in the first several $\mathrm{MeV}$ of the accelerator. The optimum transportable charge per unit volume $(\rho)$ may be considered as the figure of merit, which is proportional to $\lambda / \mathrm{p}^{2}$. Obviously, there is no limit on $\lambda$ as the channel size is increased.

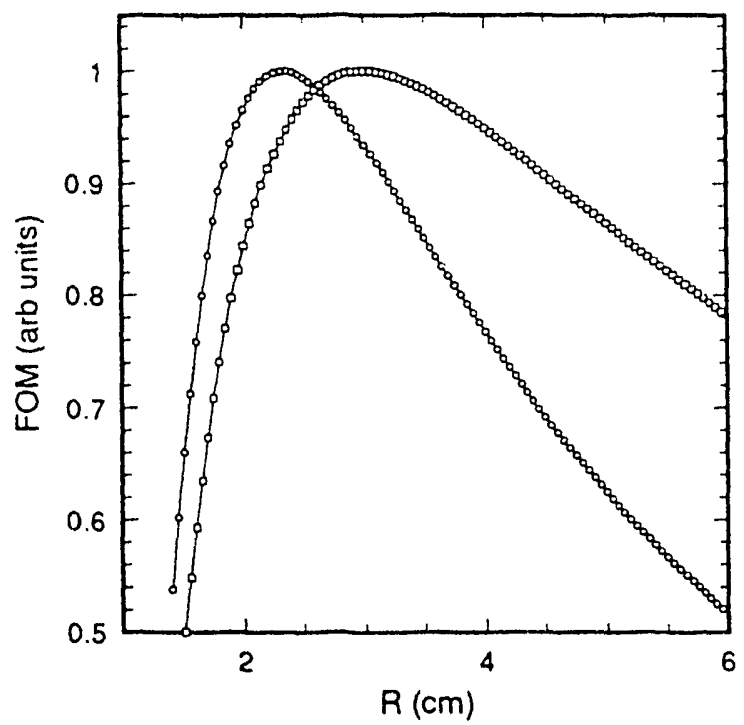

Figure 2. Figure of merit for electric focusing beam transport is proportional to $\lambda / \mathrm{p}^{2}$, shown here in arbitrary units for two functional dependences of $V_{\max }$ on the quadrupole aperture radius. The squares and the circles are for a linear and a square-root dependence, respectively.

The dependence of $V_{q}$ on $R$ has been unknown due to the complicated three-dimensional nature of the fields, the presence of insulators, and the ambiguities of the breakdown process. The vacuum breakdown dependence for simpler situations has been measured to be linear for gaps smaller than $1 \mathrm{~cm}$, and $\propto \mathrm{g}^{0.5}$ for $\mathrm{g}>5 \mathrm{~cm}$, with a transition region in between. Based partly on these simpler systems, and the clearance prescription of Eq. 2, it has long been thought that the optimum aperture radius is near $3 \mathrm{~cm}$, with an electrode to electrode gap near $2 \mathrm{~cm}$. Figure 2 illustrates the FOM for the

\footnotetext{
tThe $+1 \mathrm{~cm}$ clearance is excessive right at the entrance to the accelerator, because the coherent oscillations require some distance to develop. Therefore, the clearance could initially be decteased from that given by eq. 2 , and $\lambda$ increased.
}

linear and square root dependences. The impact on the cost of an induction linac driver is significant, since an aperture difference of $\Delta \mathrm{R}=0.6 \mathrm{~cm}$ translates into a $20 \%$ reduction in $p$, and in the overall focusing array diameter. For typical parameters this is a $15 \%$ change in the amount of induction core material needed to supply the required acceleration gradient.

The quadrupoles should be operated at approximately one-half of their breakdown voltage, $V_{\max }$, which is consistent with the operation of other high-voltage apparatus. $V_{\max }$ is not a well defined quantity because it varies with electrode materials, surface finish, contaminants and high-yoltage conditioning. For a multiple beam array nested inside the induction cores, it would not be practical to bake such a large system in situ, nor would it be desirable to heat the quadrupoles because of their precision alignment. In this application, the statistics and physics in the operation of a large array would lower the operating voltage, as would small, unavoidable beam spills.

\section{EXPERIMENTAL SETUP}

The issues of alignment tolerances and the difficulties associated with supporting the quadrupoles horizontally in an accelerator lead to consideration of hollow electrode designs and insulator supports that are not necessarily normal to the endplates or parallel to the electrodes. In order to expedite and simplify the interpretation of the results, these considerations were not included in the design of the quadrupoles tested in the experiment described here (see Fig. 3).

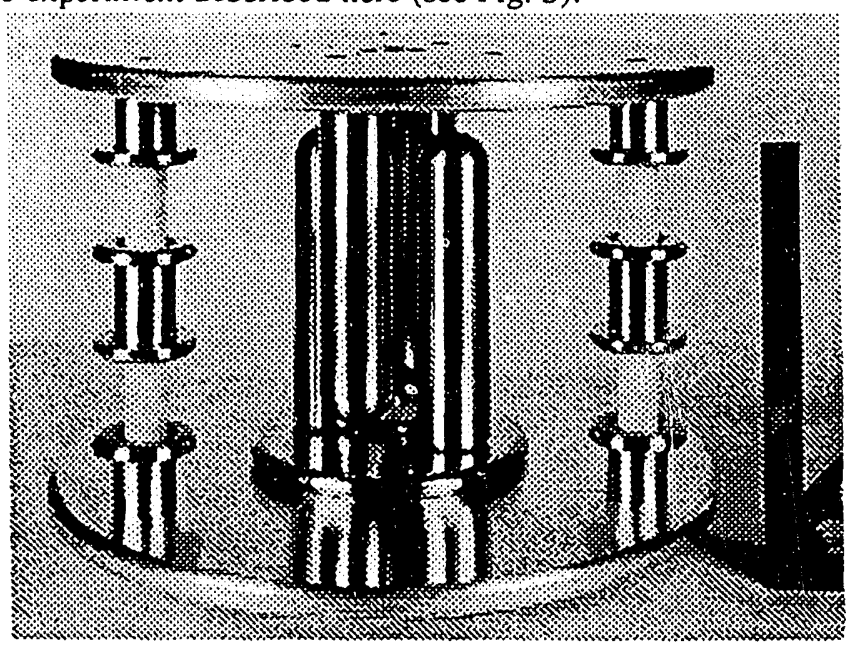

Figure 3. Quadrupole A is shown with the $R_{e l}=2.54 \mathrm{~cm}$,

$\mathrm{L}=21.5 \mathrm{~cm}$ electrodes. The scale on the right is $1 \mathrm{ft}$ high.

The goal of the experiment was to study the voltage holding characteristics of the quadrupolar electrode geometry in order to determine the optimum electrode size and spacing. Once this is established, future studies will address the issues of alignment and compact design, based on $\mathrm{V}_{\max }$ determined in this simpler experiment.

Since it was desired to have the controlling mechanism for breakdown be vacuum discharges between electrodes, the insulating supports were placed rather far away from the quadrupole electrodes. The electrodes and end-plates were solid stainless steel, and the insulating supports were made of $98 \%$ alumina $\left(\mathrm{Al}_{2} \mathrm{O}_{3}\right)$. Three sets of electrodes with hemispherical ends were constructed and arranged as described in Table I. 
Events at the interface between vacuum, dielectric, and conductor (triple-point) have been known to be controlling mechanisms for surface breakdown due to the high field concentration and field emission at the junction. To mitigate these effects, conducting shielding structures ("flower pots") were designed to reduce the field stress at the triple point. These flower pots also shielded the potentially high field region at the end of the supporting set screw that secured the insulator. For each of the three measurements, high voltage from two $(-300 \mathrm{kV} / 1.0 \mathrm{~mA}$ maximum and $+80 \mathrm{kV} / 15 \mathrm{~mA}$ maximum $)$ Glassman DC power supplies was supplied to the opposing endplates. The quadrupole rested vertically on a high-voltage feedthrough connected to the $-300-\mathrm{kV}$ power supply. The pressure in the $1 \mathrm{~m}$ diameter $\times 3 \mathrm{~m}$ long vacuum tank was measured to be $0.5-5 \times 10^{-6}$ Torr with an ionization gauge a few meters away from the quadrupole.

TABLE 1. Electrode sizes $\left(R_{e l}\right)$, spacings and voltages.

\begin{tabular}{|l|l|l|l|l|l|}
\hline Quad \# & $R(\mathrm{~cm})$ & $R_{\text {el }}(\mathrm{cm})$ & $p(\mathrm{~cm})$ & $g(\mathrm{~cm})$ & $V_{\max }(\mathrm{kV})$ \\
\hline \hline A & 2.20 & 2.53 & 6.67 & 1.63 & 230 \\
B & 3.00 & 3.44 & 9.09 & 2.22 & 270 \\
C & 3.49 & 4.00 & 10.58 & 2.58 & 289 \\
\hline
\end{tabular}

\section{RESULTS AND DISCUSSION}

The breakdown voltage for eacin quadrupole geometry was reached after about one day of pumping followed by about one-half day of conditioning, during which the vacuum system -- including the quadrupole under test -- was outgassing. Breakdown was evident by current and voltage fluctuations on the power supplies, by pressure fluctuations, concurrent visible light emission from the electrodes and fluorescence from the insulators. Slightly above $V_{\max }$, the frequency of breakdown increased sharply, while slightly below $\mathrm{V}_{\max }$ the voltage stability improved substantially. This stability was, however, not complete: occasional surface discharges were still visible from the supporting insulators below $V_{\max }$. In addition, $X$-ray production was detected helow $V_{\max }$. The source of these $\mathrm{X}$-rays is not completely clear, though it was determined to be associated with imperfections in the geometry of the $300-\mathrm{kV}$ vacuum feed-through. In fact, the voltageholding capability of the $300-\mathrm{kV}$ feed-through alone was inferior to that of the tested quadrupoles, and this enigmatic behavior is still under study. The possible impact of the flux of $\mathrm{X}$-rays on the voltage holding of the quadrupoles was a serious concern, and was studied and minimized by varying the bias between the positive and negative power supplies.

It is clear from Fig. 4 that the experimentally-determined $V_{\max }$ varies nearly as $\sqrt{g}$ in the $R=2-3 \mathrm{~cm}$ region of interest. Thus the relevant figure of merit curve has a maximum at $\mathrm{R}=2.3 \mathrm{~cm}$ in Fig. 2. Regarding the breakdown between the stainless steel electrodes, possible mechanisms of high-voltage vacuum breakdown have been discussed in the literature extensively in the past few decades, and no single mechanism has been uniquely identified as responsible. For example: electrons produced from microprotrusions on the cathode surface heat both the anode and the cathode and the melted microprotrusions may give rise to metallic ejection which could initiate a spark. Another mechanism is that the electrons bombard the anode surface producing desorbing gas which may have adverse effects on the local vacuum pressure.

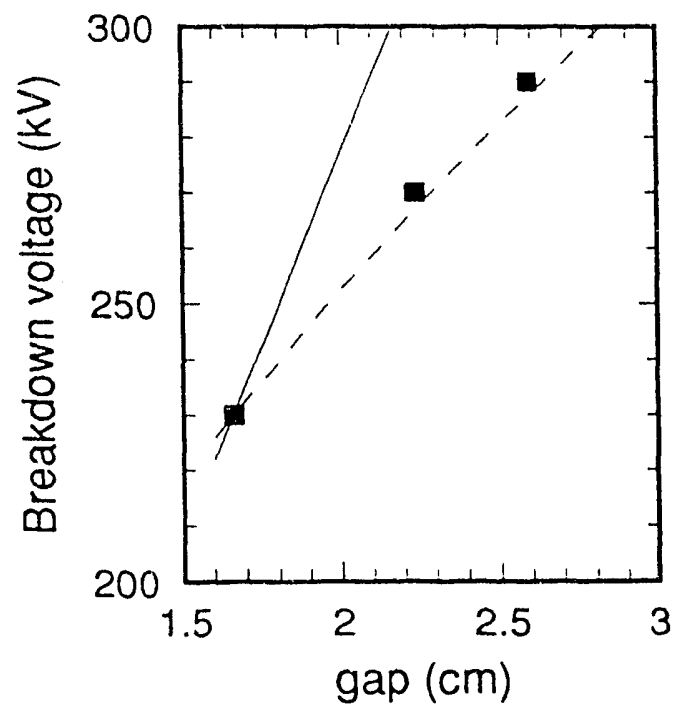

Figure 4 . The experimentally determined breakdown voltage (squares) follows a $\mathrm{g} .5$ dependence (dashed line). The solid line is a linear dependence.

In summary, we have measured the breakdown voltage vs. size relation for electrostatic quadrupoles designed for use in induction linacs for heavy ion fusion drivers. The breakdown dependence (measured in a beam-free vacuum) is proportional to $\mathrm{R}^{0.5}$ in the regio: of interesh, and the optimum aperture radius is $2.3 \mathrm{~cm}$. Assuning the aperture-filling prescription of Eq. 2, the resulting transportable charge may be estimated: Assuming that a conservative operating point is $V_{q} \equiv \pm 0.6\left(V_{\max } / 2\right)= \pm 71 \mathrm{kV}$ the corresponding line charge density approaches $\lambda=0.19 \mathrm{l} / \mathrm{C}$ at $\mathrm{T} \approx 65 \mathrm{MeV}$. Likewise, the beam volume charge density averaged over the quadrupole structure would be $\lambda / \mathrm{p}^{2}=38 \mu \mathrm{C} / \mathrm{m}^{3}$ in a multiple beam array. This does not include edge effects of electrode packing and space required for high voltage holdoff at the periphery, which are minor corrections for a large array.

\section{ACKNOWLEDGEMENTS}

The authors thank W. Tiffany, W. Ghiorso, K. Houston, C. Pike, T. Purtell, J. Rice and J. Stoker for their technical support during the experiment.

\section{REFERENCES}

[1] For another possible design see C. M. Celaıa, Proceedings 1987 Particle Accelerato: Conf., p. 996 (1987).

[2] Selected works of L. Jackson Laslett, Lawrence Berkeley Laboratory Pub-616, Vol. III, chapter 6 (1987).

[3] A. W. Maschke, Brookhaven National Laboratory BNL51029 and BNL-51419 (1979).

[4] M. G. Tiefenback and D. Keefe, IEEE Trans. Nucl. Sci., NS-32, No. 5, 2483 (1985).

[5] A. W. Maschke et al., IEEE Trans. Nucl. Sci., NS-30, No. 4, 2558 (1983). 

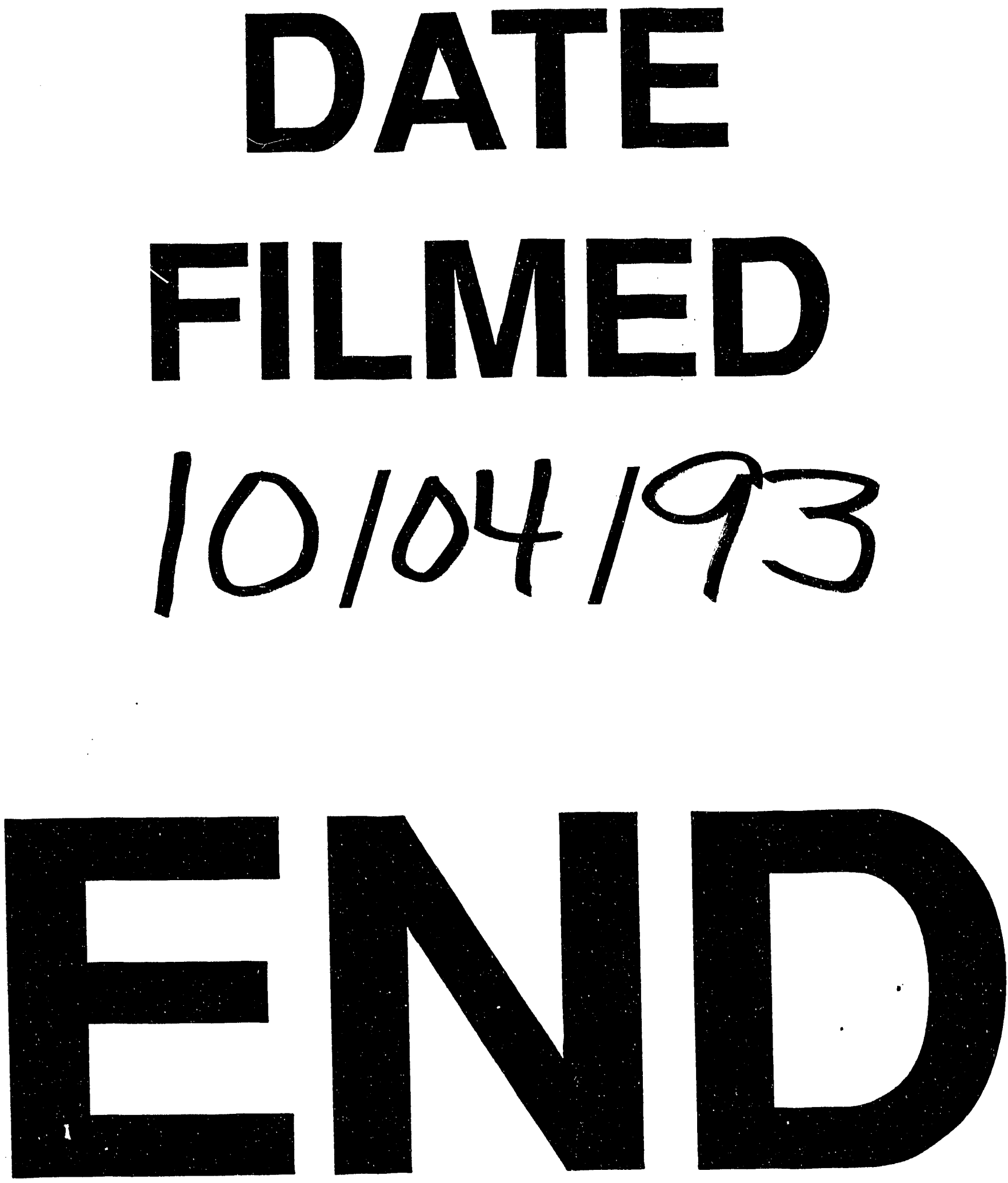
